\title{
Estilos comunicativos docentes y su incidencia en los modos de participación de los padres desde la mirada de madres de grupos vulnerables
}

\section{Teaching Communication Styles and Their Incidence in the Participation Modes of Parents From The Perspective of Mothers of Vulnerable Groups}

Verónica Gubbins*

Universidad Finis Terrae, Santiago, Chile

ORCID: http://orcid.org/0000-0003-2175-2941

\section{Elisa Ugarte}

University of California, Davis, Estados Unidos

ORCID: https://orcid.org/0000-0003-1803-9619

\section{Héctor Cárcamo}

Universidad del Bío-Bío, Chillán, Chile

ORCID: https://orcid.org/0000-0003-2045-343X

Recibido 13-11-19 Revisado 20-01-20 Aprobado 11-03-20 En línea 08-05-20

*Correspondencia

Email: vgubbins@uft.cl
Citar como:

Gubbins, V., Ugarte, E., \& Cárcamo, H. (2020). Estilos comunicativos docentes y su incidencia en los modos de participación de los padres desde la mirada de madres de grupos vulnerables. Propósitos y Representaciones, 8(2), e408. Doi: http://dx.doi.org/10.20511/pyr2020.v8n2.408 


\section{Resumen}

La experiencia subjetiva que subyace a los modos cómo las familias de grupos vulnerables participan en la escuela, tiende a invisibilizarse dentro del amplio conjunto de estudios sobre prácticas de participación de los padres en la escuela y equidad educativa. Se presentan los resultados del análisis estructural semántico de 19 entrevistas semi-estructuradas realizadas a madres de estudiantes que se desarrollan en medios socialmente desfavorecidos, orientadas a responder la pregunta de investigación siguiente: ¿cómo incide el estilo comunicativo docente, percibido por padres desfavorecidos, en su modo de participar en la escuela? Los resultados sugieren la preeminencia de un estilo comunicativo docente de carácter crítico y desvalorizador por sobre el reconocimiento hacia las competencias parentales de estas madres. Esta percepción, inhibe la participación de los padres, pero también estimula mayor vigilancia de los padres hacia el trabajo docente en el aula (v.g. voluntariado en el aula o acciones reinvidicativas del derecho a ser considerado acudiendo a otros actores con más poder dentro de la escuela). Los resultados de este estudio instan, a las políticas educativas y a los profesionales de la escuela a generar, en conjunto con sus apoderados, una cultura escolar libre de trato discriminatorio y con mayor reconocimiento a su capacidad, necesidades y posibilidades de participar en los procesos de desarrollo y aprender escolar de los hijos/as en distintas esferas de acción.

Palabras clave: Relación docente-padres; Participación de los padres; Grupos vulnerables.

\section{Summary}

The subjective experience that underlies the ways in which families underprivileged participate in school life tends to be invisiblized within the broad range of studies on parents' school involvement practices and educational equity. This article presents the results of a semantic structural analysis of 19 semistructured interviews with mothers of students living in socially underprivileged environments. The interviews were aimed at answering the following research question: "how do teacher communicative styles, as perceived by underprivileged parents, influence their way of participating at school?" Results suggest the preeminence of a critical and undervaluing teacher communicative style that prevents mothers' parental competences from being recognized. This perception inhibits parental participation while also encouraging parents to be more vigilant of teacher work in the classroom (e.g. volunteering to work in the classroom or engaging in actions aimed at defending their right to be acknowledged by appealing to actors with more power within the school). The results obtained should encourage policymakers and school professionals, along with parents, to generate a school culture that is free from discriminatory treatment and more appreciative of parents' abilities, needs, and potential for taking part in the school-based development and learning of children.

Keywords: Teacher-Parent Relationship; Parental Involvement; Underprivileged Groups.

\section{Introducción}

El estudio de la participación de los padres en la escuela ha suscitado gran interés entre investigadores educacionales de distintos países del mundo. Desde diversos enfoques teóricos y metodológicos, se ha demostrado su importancia en los procesos de mejora de la educación, desarrollo y aprendizaje escolar infantil. Aunque existe consenso respecto a la capacidad predictiva de las condiciones económicas y educacionales de origen de los estudiantes sobre la trayectoria escolar de la infancia (Hartas, 2015), varios metaanálisis de estudios -realizados preeminentemente en países anglosajones-muestran efectos aún más significativos cuando se trata de estudiantes que se desarrollan en medios socialmente desfavorecidos (Castro, Expósito, López, Lizasoain, Navarro \& Gaviria, 2015; Jeynes, 2017). 
Estos antecedentes son relevantes de ser considerados por los tomadores de decisiones vinculados a políticas educativas, gestión escolar y pedagógica de países que describen importantes diferencias en la distribución de los ingresos familiares en su población, como es el caso de muchos países latinoamericanos (World Bank, 2015). En estos países, la desigualdad socioeconómica se acompaña además de brechas significativas en los resultados que sus estudiantes obtienen en los sistemas de medición comparada de la calidad educativa a nivel internacional (OCDE, 2016). Para el caso de Chile, aunque se registran avances, los estudiantes que viven en medios socialmente desfavorecidos siguen siendo los más perjudicados (MINEDUC, 2018). La buena noticia es que la evidencia empírica regional muestra cómo el trabajo de la escuela a nivel de aula y la participación de los padres en la escuela pueden hacer una diferencia (Murillo \& Román, 2011).

En términos generales, el concepto de participación de los padres refiere a "un amplio rango de actividades y conexiones entre familias, escuelas y comunidades" (Sheldon \& Epstein, 2002, p. 197, que se despliegan en al menos tres esferas de acción: (a) en el hogar (v.g. lecturas conjuntas; apoyo en tareas escolares (b) en la escuela (v.g. asistencia a entrevistas con los docentes; reuniones de apoderados; voluntariado en la escuela) (c) la comunidad (v.g. obtención de recursos para la escuela; atención de salud para los estudiantes) (Epstein et al., 2019).

Los estudios que comparan la frecuencia de participación parental de los padres, según clase social, muestran que, no obstante que los grupos vulnerables han incorporado el credencialismo dentro de sus estrategias educativas familiares, participan menos en la escuela que los grupos socialmente más favorecidos (Gimeno \& Callejo, 2014; Gubbins \& Otero, 2016; 2018; Río Ruiz, 2010; Vincent, Rollock, Ball \& Gillborn, 2012). Se han identificado explicaciones relacionadas con el tiempo y energía de los padres para responder a las demandas de la escuela, el volumen de ingresos familiares disponibles, tipo de jornada laboral, información y comprensión de los padres respecto al sentido de participar en las actividades organizadas por la escuela, sentimientos de baja auto-eficacia parental para apoyar los procesos escolares de los hijos/as, edad y características personales de los estudiantes (Epstein et al., 2019; Gubbins \& Otero, 2018; Hoover-Demsey \& Sandler, 1995; 2005; Martín Criado, Río Ruiz \& Carvajal Soria, 2014; Reininger \& Santana, 2017) y cantidad de información que los padres reciben de parte de sus docentes (Gubbins \& Otero, 2016; Park \& Holloway, 2016; Stacer \& Perrucci, 2013). Menos conocimiento se tiene de la relación entre estilos comunicativos docentes y participación de estos padres en la escuela.

Teóricamente, el enfoque eco-relacional del desarrollo humano (Bronfenbrenner, 1979/2002), ha relevado la importancia de abordar los procesos comunicativos que se construyen en la relación entre padres-docentes. Lo que aquí se postula es que el curso del desarrollo y aprender escolar infantil no solo depende de la acción y significados que se movilizan en la cotidianeidad de la vida del estudiante en el hogar y en la escuela. Estas influencias socializadoras no se despliegan en forma separada unas de otras. El tránsito que los niños y niñas hacen, entre familias y escuela, los somete a influencias que se van superponiendo unas a otras. Especialmente durante los primeros años de escolarización. De ahí la importancia de las interacciones entre padres-docentes en la producción de ambientes educacionales potenciadores del desarrollo y aprender escolar de la infancia. Es en las interacciones que se despliegan en distintos espacios institucionales, formales (v.g. entrevistas, reuniones de padres) e informales (v.g. actividades extracurriculares, entrada y salida de estudiantes de la jornada escolar) que se entrecruzan disposiciones y prácticas que pueden conducir al reconocimiento y acciones de colaboración mutuamente acordadas, o bien, al desencuentro o el distanciamiento entre unos y otros (Miranda \& Cabello, 2016).

Autores como Hoover-Demsey y Sandler (1995) consideran que las políticas educativas, las escuelas y su profesorado podrían hacer más, en favor de la participación de los padres en la escuela, en la medida que exista una mejor comprensión de las motivaciones parentales subyacentes para participar de uno u otro modo en la escuela. Reconociendo que la escuela no tiene la capacidad de alterar las condiciones de vida de una familia, estos autores plantean que los 
docentes sí podrían intervenir en aspectos de orden subjetivo, tales como los sentimientos de autoeficacia parental para apoyar los procesos escolares de sus hijos/as, sentirse invitados a participar y acogidos por los docentes cuando así lo hacen, entre otros. Si esto es así, entonces, las políticas y acciones de mejora de la educación, en favor de estudiantes que provienen de medios socialmente desfavorecidos, tendrían más impacto si consideraran la experiencia subjetiva, necesidades y posibilidades de los padres para responder a las demandas de participación que les hace el profesorado (Wilder, 2014).

No obstante, Chile no cuenta con suficiente información que sirva de referencia para la toma de decisiones de la escuela a este respecto. Se ha profundizado poco en la comprensión de la perspectiva que padres de grupos vulnerables tienen acerca del estilo comunicativo docente y su relación con su experiencia subjetiva de participar en la escuela. Este estudio pretende contribuir a disminuir esta brecha de conocimiento abordando la pregunta ¿cómo incide el estilo comunicativo docente, percibido por padres desfavorecidos, en su modo de participar en la escuela? El objetivo de esta investigación es analizar la relación entre estilo comunicativo docente y la participación de los padres en la escuela, desde la mirada de madres de estudiantes de instituciones educativas públicas situadas en una comuna que concentra una alta proporción de familias que bien en condiciones socialmente desfavorecidas.

La noción de estilos comunicativo docente alude al conjunto de actitudes que el profesorado comunica a los padres de sus estudiantes, a través de las interacciones que establecen con aquellos en distintos espacios institucionales y, que crean un clima en el cual la información que los docentes transmiten, son interpretados por los padres, afectando el sentido de su comportamiento en la escuela. Este concepto se apoya en un enfoque interaccional-pragmático de la comunicación interpersonal (Watzlawick, Beavin \& Jackson, 1981; Bateson, 1998), el cual plantea que la comunicación humana compromete y define el tipo de relación que se construye entre los interactuantes a partir de las interpretaciones que los sujetos hacen de los contenidos percibidos y los espacios y medios utilizados para aquello (Watzlawick et al., 1981). Considera la importancia del intercambio de información y los significados que los interactuantes producen de cómo actuar en dicha relación. Como lo señala Cortés (2017), "Es la forma que adopta la comunicación y no sus contenidos, el factor más decisivo para provocar consecuencias en el comportamiento de las personas" (p.77). Este artículo plantea que el estilo comunicativo docente se convierte en un eje articulador de los modos en que los padres de grupos desfavorecidos participan en la escuela.

En varios países se debate la necesidad de cambiar la lógica asimétrica de relación padresdocentes hacia una más inclusiva y partenarial (Epstein et al., 2019; Kim \& Sheridan, 2015; McWayne, 2015). Este planteamiento ha estado siendo recogido por varias políticas educativas latinoamericanas, incluidas las de Chile (Ley No 28628, Perú; Ley General de Educación, México; Ley $\mathrm{N}^{\circ} 26206$, Argentina; Ley $\mathrm{N}^{\circ} 20370$ y Ley $\mathrm{N}^{\circ} 20845$ en Chile). No obstante, lo anterior, las recientes movilizaciones sociales que se están observando en varios países latinoamericanos, están reinvidicando el derecho de las familias de grupos vulnerables a ser reconocidos por los distintos sistemas sociales, entre otros, el sistema escolar.

Este artículo aporta evidencia que ayuda a visibilizar una dimensión que afecta el desarrollo de una cultura democrática e inclusiva y que no ha sido suficientemente problematizada a nivel de la formación inicial docente y las escuelas. Su relevancia radica en que aporta información para tomar medidas preventivas para la promoción de ambientes escolares donde prime más la lógica asociativa y partenarial por sobre aquellas más asimétrica y desvalorizadora, no obstante, las precarias condiciones económicas y culturales de las familias que pudiesen estar atravesándolos (Arón, Milicic \& Armijo, 2012; Cárcamo, 2019; Montecinos, Sisto \& Ahumada, 2010). 


\section{Método}

Los resultados que a continuación se presentan, son parte de un estudio cualitativo mayor, de carácter exploratorio, descriptivo e interpretativo (Ugarte, 2017). La investigación general estuvo orientada a analizar el rol de las familias ante la escuela, cualidad de la relación entre familias y escuela y participación parental en contextos de escuelas públicas, desde la perspectiva de las familias. El estudio se llevó a cabo en una comuna del sector suroriente de la ciudad de Santiago, Chile, donde un $24 \%$ de la matrícula de los estudiantes de la comuna es pública (municipal) (CORMUP, 2016). Las instituciones educacionales seleccionadas fueron tres escuelas públicas de enseñanza básica. Los criterios de inclusión de los participantes fueron: personas que oficien de principales responsables del cuidado del alumnado e interlocución con el profesorado que trabajan en el segundo o cuarto año nivel de educación básica (dos niveles educacionales que reciben mucha atención pedagógica y evaluativa de la calidad de su educación medida a partir de pruebas estandarizadas en Chile). El método para la selección de los participantes siguió los lineamientos de Patton (2002) conocido como purposeful random sampling. Este método propone emplear la técnica de selección aleatoria de los participantes, con el propósito de que los estudios cualitativos aseguren diversidad de perspectivas entre los hablantes entrevistados (y no la representatividad de los resultados) como es lo que se busca con el muestreo aleatorio en los estudios cuantitativos (Onwuegbuzie \& Leech, 2007). Esta técnica reduce el sesgo de autoselección de las madres que suelen ser más visibles en las actividades de la escuela (Maxwell, 2012). También permite proteger la confidencialidad de los participantes ante la escuela, cuestión que habría sido más difícil de lograr al tomar como criterio de variabilidad antecedentes académicos de los hijos e hijas, por ejemplo. La muestra quedó configurada por 19 mujeres que consintieron participar en forma voluntaria y escrita, en el estudio. No se logró obtener consentimiento de padres varones contactados. Este conjunto de madres se caracteriza por una edad promedio de 39 años; una media de 3,2 hijos; educación media incompleta promedio (solo se cuenta con un caso con educación básica incompleta y dos con educación superior completa) y un ingreso promedio familiar entre 250,000 - 350,000 CLP. En cuanto a la estructura familiar, 9 de ellas viven en pareja, 10 en hogares monoparentales. En lo ocupacional, 10 trabajan a tiempo completo, 9 trabajan a medio tiempo o son dueñas de casa.

\section{Procedimiento y técnicas empleadas}

Con la correspondiente visación ética de las autorizaciones y consentimiento informado, realizados por The Committee for Protection of Human Subjects (CPHS) de la Universidad de California, Berkeley (donde se enmarcó el estudio más amplio), se solicitó autorización escrita de la Dirección de Educación Municipal, sostenedora de los establecimientos educacionales. Inicialmente, se decidió informar al conjunto de apoderados(as) de los estudiantes de los cursos en estudio, a través de una comunicación que detallaba el objetivo y procedimientos del estudio. Luego, se procedió a realizar el contacto con las madres por vía telefónica. Una vez confirmado que se trataba de madres que ejercen rol de apoderada de sus estudiantes ante la escuela, se les explicó el contexto y objetivos del estudio. Para garantizar su aceptación voluntaria se les solicitó la firma de un consentimiento escrito y su autorización para grabar la sesión. La recolección de datos se realizó a partir de entrevistas semi-estructuradas por la oportunidad que presentan para la exploración de la subjetividad de los participantes (Maxwell, 2012). Las entrevistas se realizaron durante los meses de junio y julio del año 2016 y cada una duró aproximadamente 1 hora y 15 minutos.

\section{Método de Análisis}

El método de análisis estuvo guiado por el Método de Análisis Estructural Semántico (Greimas 1966; Piret, Nizet \& Bourgeois, 1996). Su elección se fundamenta en que permite reconstruir el mundo de significados y las valorizaciones que los sujetos construyen en los contextos culturales donde se desarrollan. El análisis se inicia con la identificación y codificación de las unidades mínimas de significado (palabras u oraciones), las cuales se organizan en términos de su valorización en oposición binaria entre sí. Estas unidades (manifiestas o implícitas) se van categorizando en ejes semánticos, en función de la valorización positiva o negativa atribuida por el hablante y los significados que las 
articulan. Este primer nivel de análisis permitió identificar dos estilos comunicativos docentes y modos diferentes de "reaccionar" a las demandas de participación de los padres en la escuela.

A continuación, el método propone indagar en un análisis de segundo orden. A saber, identificar las eventuales conexiones semánticas entre códigos de base de calificación similar o inversa. En el primer caso, se reorganizan en una nueva estructura paralela donde un código de calificación de base pasa a constituirse ahora en una nueva dimensión de análisis. En la medida que se encuentren códigos de calificación inversa, estos se organizan en estructuras cruzadas que dan pie a la construcción de campos de significado, o lo que Martinic (2006) denomina productos axiales. Cada uno de ellos condensa un conjunto de calificaciones que contribuyen a diferenciar y caracterizar distintas tipologías o perfiles de acción. En este caso, significados diferentes atribuidos a la participación en la escuela. Estos significados emergen de la conexión semántica entre estilo comunicativo docente percibido (reconocimiento y desvalorización) y modos de participar en la escuela (colaboración y repliegue).

Con el propósito de prevenir sesgos interpretativos se optó por realizar una triangulación del análisis de los datos entre dos investigadoras. Luego de la definición conjunta de los criterios para la identificación de las unidades mínimas de análisis, se prosiguió con la construcción de categorías y la elaboración de relaciones subyacentes los cuales se iban contrastando de manera sistemática, a modo de comprobar consistencia en las interpretaciones que cada una de ellas iba realizando.

\section{Resultados}

Es importante comenzar señalando que los estilos comunicativos docentes y su relación con los modos de participar en la escuela que aquí se construyeron, corresponden a un hallazgo de la investigación. Las unidades mínimas de significados que se fueron identificando y la codificación realizada a partir de las valoraciones realizadas por las entrevistadas permitieron reconocer al menos dos estilos, los cuales serán descritos con más detalle más adelante: (a) estilo crítico o desvalorizador (b) estilo orientado al reconocimiento de las capacidades de los hijos/as y el esfuerzo de participación parental. Estos estilos iban emergiendo como un hito relevante en el discurso de todas las entrevistadas, definiendo, desde la mirada de las propias participantes, distintos modos de responder o "reacciones" a las demandas de participación que les hace el profesorado. A continuación, y luego de una breve descripción de los espacios de comunicación entre docentes-familias, más y menos valorados por las participantes, exponemos cada uno de esos estilos comunicativos y su relación con distintos modos parentales de participar que se infieren del análisis de los relatos realizados.

\section{Espacios de comunicación}

En términos de los espacios institucionales de comunicación, más referenciados por las entrevistadas, solo se destacan dos. Uno más formal, como son las entrevistas personales y las reuniones de apoderados. Otros más informales, como es el encuentro entre algunos docentes y los apoderados que se reúnen a esperar a sus hijos/as a la hora de término de la jornada escolar de los estudiantes. El encuentro más personal es el espacio de comunicación más valorado, por la oportunidad de recibir información personalizada acerca de los procesos escolares de los hijos/as. No así para el caso de los lugares públicos, donde prima la percepción de quejas y críticas públicas hacia algunos estudiantes y sus padres de parte de los docentes que se encuentran de turno, cuidando la salida de los estudiantes. Como lo señala una de las madres entrevistadas:

“... ver a los niños más de manera personalizada, y ahondar un poco más en el niño. No solo porque se porte mal, el niño, no sé, es porro, no va a aprender. Entonces como que estudiar más el comportamiento del niño, ver también más a nivel familiar qué es lo que está pasando, eso" (XII, 11).

Junto a las entrevistas personales, las reuniones de padres, organizadas por los docentes, son consideradas como un espacio relevante para los padres. Es un espacio donde circula información considerada valiosa para el desempeño y logros escolares de los hijos/as. No se mencionan otros espacios de comunicación entre docentes-padres que pudiesen complementar el 
acceso y/o circulación bidireccional de información entre ambos actores, tales como correos electrónicos, Facebook o WhatsApp.

\section{Desvalorización como discurso docente dominante}

La opinión mayoritaria de estas participantes es que hay una tendencia a que los docentes se preocupen más por los problemas conductuales de los estudiantes en el aula que por su rendimiento académico o logros escolares. Esta observación se hace en referencia a la mayor convocatoria a entrevistas que los docentes les hacen a los padres con hijos/as con problemas de comportamiento en la escuela. Retomando lo planteado por una de las madres entrevistadas:

"O sea no aprendían nada, era pura citación de apoderados porque se puso a pelear...Después los cambie a otro colegio ... Pasó lo mismo..." (V, 2).

La categoría estilo comunicativo docente "desvalorizador" se construye a partir de la observación de críticas reiteradas de los docentes hacia el comportamiento de algunos estudiantes y/o la responsabilidad de sus padres a este respecto, en los horarios de salida de la jornada escolar. Estos contenidos serían transmitidos sin consideración a la presencia de uno u otro padre o madre en el lugar. Así lo detalla una de las entrevistadas:

“... si ellos ven que los niños andan tirando toda la basura en la calle, las tías le dicen "Oh que son cochinos los niños que tienen...como serán en la casa”... O si son garabateros... la mamá y el papá son garabateros" (XIII, 12).

Otras participantes, manifiestan haber observado poca consistencia en el cumplimiento de los horarios comprometidos por la institución. Se reporta que no serían pocas las veces en que la escuela decide, de manera unilateral, cuando y a qué hora termina la jornada escolar. Estas decisiones no son informadas de manera oportuna a los padres, ni son el resultado de acuerdos previos entre docentes y padres. Estas prácticas producirían sentimientos de inseguridad hacia la capacidad que la escuela tiene de asegurar cuidado y protección de sus hijos/as y poca comprensión y flexibilidad para gestionar los horarios laborales y el desplazamiento en transporte público en las grandes ciudades. Las decisiones que se toman no consideran las necesidades y posibilidades de los padres de los estudiantes.

Esta categoría, estilo comunicativo desvalorizador, también se construye a partir de unidades de significados alusivas a un exceso de importancia, percibida, de los docentes hacia la asistencia escolar de los estudiantes por sobre el rendimiento académico y logros escolares. Asistencia escolar que, para el caso de Chile, está asociado al pago de subvenciones de parte del Estado. Como lo sugiere uno de los relatos:

"Más el colegio le importa como que vayan los niños al colegio, como que vayan, que vayan, que vayan, que... más allá de lo que los niños estudien o que se superen, porque yo igual pedí hartas veces que lo metieran a reforzamiento al más grande; que lo integraran en cosas así, no lo hicieron, pero sí le importaba que el niño llegara a la hora, que el niño fuera, que fuera, que fuera, que fuera..." (III,1)

"Yo creo que no, yo creo que no la comparte, porque sabes que, encuentro que el colegio está preocupado de recibir los pagos de cada niño que les dan, tengo entendido que es así, antes que de priorizar las necesidades..." (VIII, 10)

La percepción mayoritaria es que los docentes no reconocen su buena disposición y esfuerzo para apoyar a sus hijos/as y cómo inciden, factores externos a su voluntad, en su baja frecuencia de participación en las actividades organizadas por la escuela. Se reporta poco reconocimiento a sus necesidades de contar con más información útil para apoyar a los hijos/as, tal como lo espera el profesorado. A modo de ilustración las dos citas siguientes: 
"Entonces ellos no entienden como está la situación, o sea si a los chiquillos yo los tengo así, trato en lo más mínimo que no nos falte para comer, pero estamos con lo mínimo, o sea es una cocina que me prestaron, voy a lavar a la casa de una prima de Claudio porque lavadora todavía no compramos. Entonces estamos en dos piezas, con baño incluido sí. Pero o sea mal, o sea nosotros muy apretados" (VI, 2)

“Claro porque prácticamente más que nada le echan la culpa a la mamá, que la mamá no estaba ahí, pero de repente uno nunca sabe las cosas, porque uno igual puede estar presente, pero de que realmente a su hijo uno le cuesta, le cuesta. Pero esas son las críticas que de repente dicen ..." $(\mathrm{XV}, 3)$.

\section{Hacia un estilo comunicativo docente basado en el reconocimiento}

Es interesante destacar que junto a un estilo comunicativo desvalorizador, el análisis permitió develar otro, menos visible, que hemos calificado de reconocimiento. Este segundo estilo se construye a partir de algunos de los atributos siguientes: capacidad de transmitir, de manera sincera, afecto y altas expectativas educacionales hacia los hijos/as; valorar su capacidad para aprender, habilidades y logros. Son docentes que no critican a sus estudiantes ni a sus madres en público. Por el contrario, docentes percibidos como atentos y acogedores con todos los padres, con capacidad de escucha, dar consejos y disposición a buscar soluciones junto con ellas; docentes que ocupan parte del tiempo de las reuniones de apoderados no solo en informar, sino que se detienen a explicar con más detalle aquellos asuntos de más difícil comprensión para los padres. Asimismo, son capaces de reconocer públicamente la participación de los padres sin detenerse a hacer diferencias según cuanto asisten o no a las distintas actividades organizadas por la escuela. Retomando algunas citas de lo reportado por algunas de las madres entrevistadas:

"Que siento que me lo aceptan mucho, de verdad, que no están como "oh ya tu hijo de nuevo se portó mal" "oh tu hijo..." no son puros reclamos en realidad, sino que también lo elogian mucho" (XII, 4).

"Que somos buenos apoderados, que siempre estamos ahí, unos más que otros. Pero ella siempre dice, pero ella trata como de unir, trata de explicar a los apoderados en la reunión. Si la única instancia en que se pueden juntar todos, es en la reunión, pero no todos. Pero ella siempre dice, aquí todos quienes saben, son buenos apoderados. Ella se lleva bien con todos. Les tiene una paciencia, es como bien calmaiiita" (XVI, 3).

\section{¿Colaborar o resistir?}

El análisis de las unidades de significado permitió distinguir otros dos ejes semánticos referidos, esta vez, a modos diferentes de responder a las demandas de participación que realiza la escuela: disposición a colaborar y resistencia a responder a las demandas de la escuela.

No obstante, esta disposición "colaborativa" se expresa en prácticas diferentes. Por un lado, refiere al conjunto de acciones que van del rango inicial de asistir en forma sistemática a las actividades institucionales organizadas por la escuela (v.g. entrevistas y reuniones de apoderados) hasta realizar voluntariado en actividades pedagógicas de aula. Por otro lado, de resistencia a las demandas de la escuela, manifestando disposiciones de colaboración selectiva, sea, asistiendo solo a algunos espacios convocados por la institución, preeminentemente las reuniones de apoderados, o bien dejar de hacerlo y concentrar su acción colaborativa a las actividades escolares de los hijos/as desde el hogar.

\section{Estilos comunicativos docentes y modos de participación de los padres: significados subyacentes}

Al indagar en las conexiones semánticas entre estilos comunicativos y modos de enfrentar las demandas de participación, se pudo construir una estructura cruzada, describiendo cuatro campos de significados diferentes subyacentes a los modos de participación de estas madres: (1) colaboración incondicional a las necesidades de la escuela o alianza (2) pseudo-colaboración (3) 
colaboración vigilante del trabajo docente (4) repliegue hasta conducir a la salida de la escuela. Son estos los campos los que pasamos a describir con más detalle a continuación.

\section{Disposición a colaborar en la escuela}

En primer lugar, y a partir de la conexión semántica entre estilo comunicativo de reconocimiento y disposición colaborativa a participar en las actividades formales de la escuela, emerge lo que hemos denominado "colaboración incondicional a las necesidades de la escuela" o "alianza". Este campo expresa disposición de las madres a asistir en forma regular a las actividades institucionales y colaborar en forma voluntaria en distintas actividades pedagógicas o recreativas organizadas por la escuela. Algunas participantes reportan llegar al extremo de sacrificar tiempo personal y familiar para hacerlo. Esta alianza también se expresa en una fuerte dependencia de estas madres hacia la opinión y orientaciones que reciben de los profesionales de la escuela. Siguiendo lo planteado por una de las madres:

"Porque yo se lo propuse a la profesora. Porque ella me decía que el XXX no me quiere escribir y no me quiere trabajar en el colegio. Y yo le dije "No hay problema. Si usted me da permiso yo vengo a tomar clase... Apoyé en una actividad que estaban haciendo. Puse un grupito como de 10 niños "Vengan, vamos a hacer la actividad". Y eso" (IX, 3).

\section{Participación pseudo-colaborativa}

En la medida que la desvalorización docente se hace repetitiva en el tiempo, y se recibe reconocimiento en situaciones excepcionales, algunas madres se van distanciando de las demandas de los docentes. Se mantiene presencialidad en algunas actividades de la escuela (especialmente en las reuniones de apoderados), pero la experiencia subjetiva es de distanciamiento psicológico o pseudo-participación. Se mantiene el interés por mantenerse informada de los asuntos escolares que pudiesen afectar a los hijos/as. Se escucha lo que se tiene que decir, pero son ellas las que deciden luego qué y cómo hacerlo. De este modo, y no teniendo posibilidades de optar por otro establecimiento, se opta por autoexcluirse, no respondiendo a las demandas de la escuela. Para ejemplificar, la cita siguiente:

“...mire, yo cuando voy a los colegios, yo sé a lo que voy. A escuchar puras...lo mismo de siempre. Entonces yo siempre voy a la defensiva. ¿Y a quien voy a defender? A mis hijos. Me digan lo que me digan. Me digan que mi hija se portó mal, que no sé qué...” (IV, 18).

\section{Colaboración vigilante del quehacer docente}

Al cruzar el eje "estilo comunicativo desvalorizador" con "disposición a colaborar" emergen significados que dan cuenta de valoración a la colaboración de los padres con las actividades de la escuela, pero desde una actitud más bien "vigilante" del trabajo docente en el aula. No obstante, cumplir con las demandas de participación en actividades institucionales, el análisis devela que la participación colaborativa no se acompaña necesariamente de confianza parental hacia el trabajo de los docentes. Trasunta la necesidad de un mayor involucramiento en las actividades de la escuela desde la figura del voluntariado, como "reacción" a estilos comunicativos docentes percibidos como desvalorizadores. La colaboración se orienta más a "vigilar" el actuar docente en el aula, que a constituirse en una práctica adyuvante del trabajo educativo de la escuela. Esta disposición emerge, particularmente cuando se sospecha que la escuela oculta información relevante que podría perjudicar a alguno de sus hijos/as. El único modo de informarse pareciera ser el involucramiento presencial en las actividades docentes de sala de clase. Así lo expresa una de las madres entrevistadas:

"Estuve el año pasado viniendo todos los días lunes a ayudar a los profesores, porque habían dos profesoras que los chicos no las dejaban hacer clases, y yo mi día libre me lo tomaba y venía a hacer clase con las profesoras todo el día. Desde las ocho de la mañana hasta la una de la tarde (...) es que lo que pasa es que aquí hay unas profesoras que les dan lo mismo ir a hacer clases o no hacer clases, y los chicos igual, a los chicos les daba lo mismo que fuera esa 
profesora o no fuera, porque las profesoras se sentaban, chateaban, "ya hagan esto", los chicos no lo hacían, les ponían mala nota, no hacían nada" (XVII, 11).

Frente a la percepción de resistencia o falta de compromiso de los docentes hacia las necesidades educativas de los hijos/as y poca acogida a sus propuestas de mejoramiento realizados a los docentes, algunas madres (aunque minoritarias) deciden buscar por sí mismas las respuestas a sus inquietudes en otros actores de la escuela. Unas se dirigen a otros profesionales percibidos como más abiertos y acogedores como son los orientadores y los psicólogos. Otras, acuden a la dirección del colegio para exigir soluciones a sus demandas. Frente a la desvalorización, buscan por sí mismas ser acogidas y/o encontrar respuestas a sus necesidades o la de sus hijos/as en otros actores de la escuela. Esta disposición se conecta con argumentos relacionados con la importancia de proteger los derechos de los hijos/as necesarios de ser respetados por la escuela y sus docentes:

“... porque yo vivo...(en) la Pincoya... Y ahí hay excelentes mamitas. Hay madres que vienen de abajo y crían a un buen hombre. Y salen buenos hombres. No todos son malos. Pero aquí, aquí en Chile, ponen...te digo a ti...van como discriminando. No van al mismo nivel educacional los que te dan aquí y los que te dan allá ...tiene que ser algo equitativo, para todos igual. Porque si una profesora de Las Condes no puede dar clase allá abajo. Somos todos iguales. Todos tienen el mismo derecho. Aunque los papás no te den la oportunidad de hacer una carrera o ser profesional, no podemos culpar a los niños por eso (...) Lo que sí que ... los colegios hagan talleres. Los colegios pueden hacer talleres a los papitos" (IX, 6).

Este empoderamiento no implica necesariamente adoptar conductas agresivas. Estas madres manifiestan en forma explícita la relevancia de expresarse de manera adecuada y el poder que el buen trato trae consigo a la hora de enfrentar problemas con una autoridad.

"Siempre he dicho las cosas que tengo que decir, pero siempre con respeto hacia los demás. El mismo respeto que pido que me tengan a mi, el mismo respeto que pido que le tengan con mis hijos. El mismo respeto que les pido a mis hijos que tengan con la maestra" (XIV, 6).

\section{Involucramiento parental desde el hogar o repliegue}

La percepción reiterada de actitudes docentes de desvalorización también pareciera incidir en una resistencia tal que, algunas madres, decidan replegarse dejando de asistir a las actividades organizadas por la escuela. La disposición colaborativa se desplaza desde las actividades de la escuela hacia las necesidades y actividades escolares de los hijos/as. La participación se despliega en la esfera privada del hogar y se desarrolla siguiendo los criterios que se construyeron de la experiencia escolar propia o familiar:

"Porque me aburrí, porque mi hija pasaba suspendida, porque el inspector lo único que quería era que yo la sacara del colegio. Y mi aburrí po'. Mi hija ya no quiere ir al colegio. Por lo mismo. Porque hay profesores que te...te los terminan estigma...estig (3) (...) Entonces qué es lo que pasa que ya después pasa a ser como rutina la cuestión. O sea, podí juntarte con miles de cabros pero la cagá se la manda tu hija. Entonces ya al final que "¿Saben qué?

Váyanse a la punta del cerro". No, ya no voy. O sea, de hecho me ha llamado la asistente social varias veces. No voy $(I V, 4)$...Porque aquí estoy sola. Y estoy chata, estoy aburrida. No voy a los colegios. Me llaman, no voy. No he ido a los hospitales a hacer los tratamientos que tienen que hacerse. No, estoy....estoy choreá. Estoy agotá. Y más encima los colegios lo único que hacen es criticarte. Nada más po" (IV, 12).

En situaciones extremas, este repliegue puede conducir a optar por la salida del estudiante de la escuela, buscando otras instituciones educativas donde éste pueda recibir mejor trato y oportunidades educacionales. 


\section{Conclusiones}

Siguiendo lo planteado por otros autores, los resultados de esta investigación muestran que el grado y formas de participación de los padres en la escuela se relaciona con las experiencias subjetivas que se construyen en la relación padres-docentes (Berkowitz, Astor, Pineda, DePedro, Weiss \& Benbenishty, 2017; Valdés Cuervo \& Sanchez, 2016). El estilo comunicativo docente, percibido por los padres de grupos vulnerables, construye experiencias subjetivas diversas que se relacionan con prácticas diferentes, para responder a las demandas de participación en la escuela. Estas prácticas van desde la participación presencial en actividades formales de la escuela, el voluntariado, pero también el repliegue e incluso la decisión de salida del estudiante de la escuela. El enfoque interaccional-pragmático de la comunicación sostiene la importancia de poner atención a las consecuencias del acto y estilo comunicativo sobre la conducta de los interactuantes (Cortés, 2017). El estilo comunicativo docente podría ser parte de las explicaciones de la baja participación de los padres de grupos desfavorecidos en la escuela.

Westergard y Galloway (2010) señalan que cuando “... los padres intentan establecer una relación con los docentes, sea formal o informal, pero sienten que no puede establecerse una relación de colaboración con ellos o sienten que esa relación se ha roto" (traducción personal, p. 98) las familias se desencantan y se distancian de la escuela. Un hallazgo de esta investigación es que el desencanto parental no siempre conduce al repliegue o la salida de los estudiantes de la escuela (Arón, Milicic \& Armijo, 2012; Cárcamo, 2019; Retuert y Castro, 2017; Quiocho \& Daoud, 2006; Walker, 2011). Los resultados muestran que algunas madres construyen caminos diferentes. Algunas de ellas buscarán aliarse con los docentes, como es el caso de aquellas madres que se identifican con sus necesidades y desarrollan prácticas de voluntariado en el aula (Epstein, 2013). Otras, desde un interés reinvidicativo y protección de los derechos de los hijo/as, buscan incidir en las decisiones y acciones de la escuela contactando a otros actores (v.g. psicólogos, orientadores) o autoridades del establecimiento educacional (Epstein et al., 2019). Se puede concluir que el estilo comunicativo docente percibido inhibe la participación de los padres, pero también puede estimular mayor vigilancia de los padres hacia el trabajo docente en el aula (v.g. voluntariado en el aula o acciones reinvidicativas del derecho a ser considerado acudiendo a otros actores con más poder dentro de la escuela).

El fuerte impulso dado por las políticas de inclusión escolar y no discriminación, los últimos años, han afectado en forma positiva los modos de relación entre docentes y padres de grupos vulnerables (Cárcamo-Vásquez \& Rodríguez-Garcés, 2015; Montecinos, Sisto \& Ahumada, 2010). Están cambiando los paradigmas docentes centrados en el déficit, desafección y falta de responsabilidad de estas familias (Holloway \& Kunesh, 2015; Lareau \& Colarco, 2012; Río Ruiz, 2010; Valencia, 2012). Los resultados que aquí presentamos sugieren la emergencia de una mirada nueva, en los docentes, capaz de reconocer que estos padres cuentan con recursos psicosociales para generar las mejores condiciones de desarrollo y aprender escolar para sus hijos/as. Analizar las causas posibles de la persistencia de un paradigma desvalorizador en los docentes escapa a los objetivos de esta investigación, solo podemos concluir, por el momento, que los grupos vulnerables también comienzan a relacionarse de modo distinto con la escuela. Nos enfrentamos hoy a una sociedad más letrada, tecnologizada, interconectada a nivel global y cada vez más diversa. La población se hace más vigilante, también hacia el trabajo escolar de los docentes.

No obstante, estos cambios no parecen ser percibidos por muchos docentes. La perspectiva mayoritaria, de las madres entrevistadas en este estudio, es que aún prima una mirada crítica y desvalorizadora hacia los padres de grupos vulnerables. Los docentes no pueden olvidar que existen razones externas a la voluntad de los padres, no alterables por la escuela o las propias familias, que harán siempre más difícil la participación en la escuela de unas familias respecto de otras (v.g. exigencias laborales, tiempo de desplazamiento en las grandes ciudades). Los resultados de esta investigación sugieren, en consecuencia, que este impulso político y económico en favor de una cultura escolar más inclusiva y democrática debe incluir la relación entre docentes-padres. Estudios hechos por Hoover-Dempsey y Sandler $(1995 ; 2005)$ sostienen que la percepción de sentirse invitados por el 
profesorado aumenta la participación de los padres en la escuela. Esto desafía a todos los actores educativos de la escuela a construir relaciones basadas en el buen trato y la no discriminación hacia las familias más desfavorecidas. A partir de ahí, aprender a desarrollar, en conjunto con estos padres, relaciones más cercanas a la lógica partenarial que permita incorporar el principio de la diversidad también en los modos como se definen las acciones de participación de las familias. Ampliar el campo de alternativas de la acción parental, sea en la escuela o en otras esferas de la vida del estudiante como es su hogar (Epstein et al., 2019) de manera de hacerla más efectiva y viable.

Otro hallazgo, interesante de ser destacado en este estudio, es la ausencia de referencia a procesos colectivos de mejoramiento educativo. La mirada de las madres se centra prioritariamente en el desarrollo y aprender escolar de los propios hijos/as. El principal motor de la participación de estas madres es el éxito escolar y no la equidad educativa. Prima una visión individualista de la relación con la escuela y sus docentes. Existen antecedentes que sugieren que la participación institucionalizada y representativa de los padres, vía centros de padres y consejos escolares, genera un efecto colectivo de bien común. La sumatoria de participación individual de los padres no resulta suficiente. La participación organizada de los padres, al menos para el caso de Chile, no es obligatoria. Si la escuela quiere obtener más beneficios de la participación de los padres en la escuela, debiera poder formalizar una mejor acogida a sus necesidades y posibilidades. La organización de los padres aparece como un interesante recurso para estos efectos.

La literatura consultada muestra que, y no obstante valorar la importancia de la participación parental en la escuela, los directivos y docentes no manejan suficiente información acerca de los aspectos subyacentes que motivan o no la participación de los padres en la escuela (Cárcamo, 2019; Rivera \& Milicic, 2006; Westergard \& Galloway, 2010). Los resultados de este estudio instan, a las políticas educativas y a los profesionales de las escuelas a revisar sus prácticas y estilos comunicativos y analizar cómo ello se conecta con el grado y modos de participación de los padres en y fuera de la escuela. Con especial atención en escuelas que atienden familias más desfavorecidas en lo económico y lo cultural. Asimismo, invitan a analizar los programas de formación inicial de los docentes y promover el diseño de programas de formación continua en el área de las relaciones y comunicación entre docentes-padres en distintos contextos institucionales y, revisitar, las políticas y programas de convivencia escolar de manera de integrar la relación y comunicación entre docentes-apoderados como otra dimensión relevante de considerar en los planes y programas de mejoramiento de la inclusión escolar y equidad educativa (Ley $\mathrm{N}^{\circ} 20845$ ).

Finalmente, interesa dejar consignada una "reacción" emergente que hemos denominado sobre-identificación, por sus implicancias en los procesos de cohesión interna e inclusión escolar, particularmente en aquellas instituciones educativas que trabajan con alta proporción de familias de grupos vulnerables. No obstante, vivir en condiciones de precariedad similar a otras familias del curso de sus hijos/as, algunas madres se "desmarcan" de los otros padres del curso, reproduciendo una actitud desvalorizadora hacia los pares que parecen estar más distanciados de la expectativa docente. Es una reacción que busca, en forma no consciente, el reconocimiento docente en un contexto institucional más bien crítico de las competencias de los padres de grupos vulnerables, pero que, a la vez, no contribuye a fortalecer la confianza inter-pares para un trabajo colectivo y organizado en favor de la equidad educativa de todos los estudiantes del curso. Retomando las palabras de la madre en mención:

"Porque hay reuniones que van poquísimos apoderados... O sea, puedo faltar, según yo, a una, pero no faltar todo el año. Apoderados que, por ejemplo, hay niños de la edad de XXX que no van al colegio porque hace frío... Pero, ¿Y si llueve una semana completa? ¿Y si llueve un mes completo? ¿No me va mandar nunca al colegio? Pero cuando tenga que trabajar, va a tener que ir a trabajar igual, con lluvia o sin lluvia, con barro o sin barro... Entonces, aquí hay apoderados que no mandan a los niños, porque hace frío. Y no los mandan durante varios días, y después están reclamando en las reuniones y ¿qué reclaman?” (XIV, 7). 
Finalmente, queremos recordarle al lector que los resultados del análisis que aquí comunicamos fueron emergiendo en el contexto de una investigación mayor, por lo que se sugiere considerar la hipótesis relacional entre estilos comunicativos docentes y tipologías de participación que aquí se propusieron, solo como tal. Es decir, una hipótesis interpretativa que requiere seguir siendo indagada en futuras investigaciones cualitativas y otras de mayor alcance en contextos institucionales similares y contrastantes entre sí (McWayne, 2015).

\section{Referencias}

Aron, A. M., Milicic, N., \& Armijo, I. (2012). Clima social escolar: una escala de evaluaciónEscala de Clima Social Escolar, ECLIS. Universitas Psychologica, 11(3), 803-813.

Bateson, G. (1998). Pasos hacia una ecología de la mente. Buenos Aires: Editorial Lúmen.

Berkowitz, R., Astor, R. A., Pineda, D., DePedro, K. T., Weiss, E. L., \& Benbenishty, R. (2017). Parental involvement and perceptions of school climate in California. Urban Education, 8(3), 1-31. doi: https://doi.org/10.1177/0042085916685764

Bronfenbrenner, U. (1979/2002). La ecología del desarrollo humano. Barcelona, España: Paidós. Cabello, S. A., \& Miranda, J. G. (2016). El papel y la representación del profesorado en la participación de las familias en la escuela. Revista electrónica interuniversitaria de formación del profesorado, 19(1), 61-71.

Cárcamo-Vásquez, H., \& Rodríguez-Garcés, C. (2015). Rol parental educativo: aproximación a las percepciones que poseen los futuros profesores. Educación y Educadores, 18(3), 456470. doi: https://doi.org/10.5294/edu.2015.18.3.5

Cárcamo, H. (2019). Relación familias-escuela y procesos de formación ciudadana. En Orellana, Salazar y Hasse (Eds.). Formación ciudadana en el contexto escolar. conceptualización, avances y experiencias. (pp. 29-46). Santiago: RIL.

Castro, M., Expósito-Casas, E., López-Martín, E., Lizasoain, L., Navarro-Asencio, E. \& Gaviria, J. L. (2015). Parental involvement on student academic achievement: A metaanalysis. Educational Research Review, 14, 33-46.

CORMUP. Corporación Municipal de Peñalolén (2016). Plan de Desarrollo Municipal 2016: Dirección de Educación. Recuperado de http://www.cormup.cl/wpcontent/uploads/2015/11/PADEM-2016.pdf

Cortés, C. (2017). Teoría de la Comunicación Humana. Quito: Editorial Razón y Palabra Domínguez, R. S., Castro, S. B. E., \& Cuervo, A. A. V. (2017). Participación de los padres en la educación: una prueba del modelo de Hoover-Dempsey y Sandler. Perspectiva Educacional, 56(2), 139-153.

Epstein, J. 2013. Programas efectivos de involucramiento familiar en las escuelas. Santiago: Fundación CAP.

Epstein, J. L., Sanders, M. G., Simon, B. S., Salinas, K. C., Jansorn, N. \& Van Voorhis, F. (2019). School, family, and community partnerships: Your handbook for action. Fourth edition. Thousand Oaks, CA: Corwin Press.

Gimeno, R. M., \& Callejo, C. M. B. (2014). La evolución de la importancia del capital escolar en la clase obrera. Revista de Sociología de la Educación-RASE, 7(2), 373-394.

Greimas, A. J. (1966). Eléments pour une théorie de l'interprétation du récit mythique. Communications, 8(1), 28-59. doi: https://doi.org/10.3406/comm.1966.1114

Hartas, D. (2015). Patterns of parental involvement in selected OECD countries: cross-national analyses of PISA. European Journal of Educational Research, 4(4), 185-195. doi: https://doi.org/10.12973/eu-jer.4.4.18

Holloway, S. D., \& Kunesh, C. E. (2015). Cultural processes and the connections among home, school, and community. In Processes and pathways of family-school partnerships across development (pp. 1-15). Springer, Cham. 
Hoover-Dempsey, K.V., \& Sandler, H.M. (1995). Parental involvement in children's education: Why does it make a difference? Teachers College Record, 97(2), 310-331. doi: https://doi.org/10.3102/00346543067001003

Hoover-Dempsey, K. V., Walker, J. M., Sandler, H. M., Whetsel, D., Green, C. L., Wilkins, A. S., \& Closson, K. (2005). Why do parents become involved? Research findings and implications. The elementary school journal, 106(2), 105-130

Jeynes, W. H. (2017). A meta-analysis: The relationship between parental involvement and Latino student outcomes. Education and Urban Society, 49(1), 4-28.

Kim, E. M., \& Sheridan, S. M. (2015). Foundational aspects of family-school connections: Definitions, Conceptual frameworks, and research needs. In S. M. Sheridan y E. M. Kim (Eds.), Foundational aspects of family school partnership research. (pp. 1-14). New York, NY: Springer International Publishing.

Lareau, A., \& Colarco, J. M. (2012). Class, cultural capital, and institutions: The case of families and schools. Facing social class: How societal rank influences interaction, 61-86.

Ley 26.206. Ley Educación Nacional Argentina. Recuperado de https://www.argentina.gob.ar/validez-nacional-de-titulos/ley-de-educacion-nacionalndeg-26206

Ley $\mathrm{N}^{\circ}$ 20370. Ley General de Educación. Chile. Recuperado de https://www.ayudamineduc.cl/ficha/lge-ley-general-de-educacion-4

Ley $\quad \mathrm{N}^{\circ}$ 20845. Inclusión Escolar. Chile. Recuperado de http://www.rmm.cl/bibliotecadigital/ley-ndeg-20845-inclusion-escolar

Ley $\quad \mathrm{N}^{\circ} 28628$. Ley que regula la participación de las asociaciones de padres de familia en las instituciones educativas públicas. Recuperado de https://www.gob.pe/institucion/minedu/normas-legales/118389-28628.

Martín Criado, E., Río Ruiz, M. Á., \& Carvajal Soria, P. (2014). Prácticas de socialización y relaciones con la escolaridad de las familias más alejadas de la norma escolar. Revista de la Asociación de Sociología de la Educación, 7(2), 429-448.

Maxwell, J. A. (2012). The Importance of Qualitative Research for Causal Explanation in Education. Qualitative Inquiry, 18(8), 655-661. doi: https://doi.org/10.1177/1077800412452856

McWayne, C. M. (2015). Family-School Partnerships in a Context of Urgent Engagement: Rethinking Models, Measurement, and Meaningfulness. In S. M. Sheridan \& E. M. Kim (Eds.), Foundational aspects of family school partnership research (pp. 105-124). New York, NY: Springer International Publishing.

MINEDUC (2019). Resultados educativos 2018. Recuperado de http://archivos.agenciaeducacion.cl/Conferencia_EERR_2018.pdf

Montecinos, C., Sisto, V., \& Ahumada, L. (2010). The construction of parents and teachers as agents for the improvement of municipal schools in Chile. Comparative Education, 46(4), 487-508. doi: https://doi.org/10.1080/03050068.2010.519481

OCDE (Organisation for Economic Cooperation and Development). (2016). Low Performing Students: Why They Fall Behind and How to Help Them Succeed doi: http://dx.doi.org/10.1787/9789264250246-en

Onwuegbuzie, A. J., \& Leech, N. L. (2007). Sampling Designs in Qualitative Research: Making the Sampling Process More Public.The Qualitative Report, 12(2), 238-254.

Park, S., \& Holloway, S.D. (2016). The effects of school-based parental involvement on academic achievement at the child and elementary school level: A longitudinal study. The Journal of Educational Research, 110(1), 1-16. doi: https://doi.org/10.1080/00220671.2012.667012

Patton, M. Q. (2002). Qualitative evaluation and research methods. Qualitative Evaluation and Research Methods. doi: https://doi.org/10.1002/nur.4770140111

Piret, A., Nizet, J., \& Bourgeois, E. (1996). L'analyse structurale: une méthode d'analyse de contenupour les sciences humaines. Bruxlelles: De Boeck Supérieur Université.

Quiocho, A. M., \& Daoud, A. M. (2006). Dispelling myths about Latino parent participation in schools. The Educational Forum, 70(3), 255-267. 
Reininger, T., \& Santana López, A. (2017). Parental involvement in municipal schools in Chile: Why do parents choose to get involved? School Psychology International, 38(4), 363379.

Retuert Roe, G., \& Castro, P. J. (2017). Teorías subjetivas de profesores acerca de su rol en la construcción de la convivencia escolar. Polis (Santiago), 16(46), 321-345. doi: https://doi.org/10.4067/s0718-65682017000100321

Río Ruiz, M. Á. (2010). No quieren, no saben, no pueden: Categorizaciones sobre las familias más alejadas de la norma escolar. RES. Revista española de sociología, 14, 85-105.

Rivera, M., \& Milicic, N. (2006). Alianza Familia-Escuela: Percepciones, Creencias, Expectativas y Aspiraciones de Padres y Profesores de Enseñanza General Básica. Psykhe (Santiago), 15(1), 119-135. doi: https://doi.org/10.4067/S0718-22282006000100010

Stacer, M. J., \& Perrucci, R. (2013). Parental involvement with children at school, home, and community. Journal of Family and Economic Issues, 34(3), 340-354.

Sheldon, S. B., \& Epstein, J. L. (2002). Improving student behavior and school discipline with family and community involvement. Education and urban society, 35(1), 4-26.

Torrecilla, J.F. \& Román, M. (2011). ¿La escuela o la cuna? Evidencias sobre su aportación al rendimiento de los estudiantes de América Latina. Estudio multinivel sobre la estimación de los efectos escolares. Profesorado. Revista de Currículum y Formación de Profesorado, 15(3), 27-50.

Ugarte, E. (2017). Between the Estar Encima and Estar Ahí: Chilean Low-Income Mothers Conceptualizations of Parental Engagement. Unpublished Manuscript.

Valdés Cuervo, Á. A., \& Sánchez, P. A. (2016). Las creencias de los docentes acerca de la participación familiar en la educación. Revista electrónica de investigación educativa, $18(2), 105-115$.

Valencia, R.R. (2012). The Evolution of Deficit Thinking: Educational Thought and Practice. London and New York: Routledge Farmer.

Vincent, C., Rollock, N., Ball, S., \& Gillborn, D. (2012). Being strategic, being watchful, being determined: Black middle-class parents and schooling. British Journal of Sociology of Education, 33(3), 337-354.

Walker, K. L. (2011). Deficit Thinking and the Effective Teacher. Education and Urban Society, 43(5), 576-597. doi: https://doi.org/10.1177/0013124510380721

Watzlawick, P., Beavin, J. y Jackson, D. (1981): Teoría de la Comunicación Humana. Barcelona: Herder.

Westergård, E., \& Galloway, D. (2010). Partnership, participation and parental disillusionment in home-school contacts: a study in two schools in Norway. Pastoral care in Education, 28(2), 97-107. doi: https://doi.org/10.1080/02643944.2010.481309

Wilder, S. (2014). Effects of parental involvement on academic achievement: a meta-synthesis. Educational Review, 66(3), 377-397. doi: https://doi.org/10.1080/00131911.2013.780009

World Bank. (2015). World Development Indicators 2015. Washington, DC: World Bank.

Nota: Queremos agradecer el apoyo del Programa Fondecyt/Conicyt al Proyecto No 11150098 y al programa de Human Development de la Graduate School of Education de la University of California, Berkeley. En forma especial a Susan Holloway por la oportunidad de conversar acerca de los supuestos teóricos y metodológicos que se encuentran a la base de esta investigación. La coautora agradece el apoyo de Becas Chile/CONICYT N ${ }^{\circ} 73160367$, Center for Latinamerican Studies y Tinker Foundation por el apoyo para la recolección de los datos de esta investigación para la obtención del grado de Master of Arts in Human Development and Education de la Univerity of California, Berkeley. 
